\title{
sciendo
}

\author{
BULGARIAN ACADEMY OF SCIENCES
}

CYBERNETICS AND INFORMATION TECHNOLOGIES • Volume 19, No 1

Sofia $2019 \quad$ Print ISSN: 1311-9702; Online ISSN: 1314-4081

DOI: $10.2478 /$ cait-2019-0010

\section{Optimum Design of CDM-Backstepping Control with Nonlinear Observer for Electrohydraulic Servo System Using Ant Swarm}

\author{
Fouad Haouari ${ }^{1}$, Nourdine Bali ${ }^{2}$, Mohamed Tadjine ${ }^{1}$, Mohamed Seghir \\ Boucherit $^{1}$ \\ ${ }^{1}$ Department of Electrical Engineering, Process Control Laboratory, ENP 10 Avenue Hassan Badi P.O. \\ Box 182, 16200 Algiers, Algeria \\ ${ }^{2}$ Electrical Engineering and Computing Faculty, USTHB P.O. Box 32, El Alia, 16111, Bab Ezzouar \\ Algiers, Algeria \\ E-mails:haouari_fouad@yahoo.fr bali_nordine@yahoo.fr tadjine@yahoo.fr \\ ms_boucherit@yahoo.fr
}

\begin{abstract}
This paper introduces an application of an Ant Colony Optimization algorithm to optimize the parameters in the design of a type of nonlinear robust control algorithm based on coefficient diagram method and backstepping strategy with nonlinear observer for the electrohydraulic servo system with supply pressure under the conditions of uncertainty and the action of external disturbance. Based on this model, a systematic analysis and design algorithm is developed to deal with stabilization and angular displacement tracking, one feature of this work is employing the nonlinear observer to achieve the asymptotic stability with state estimations. Finally, numerical simulations are given to demonstrate the usefulness and advantages of the proposed optimization method.
\end{abstract}

Keywords: Ant colony optimization, Coefficients diagram method, Backstepping control, Electrohydraulic servo systems, Observers.

\section{Introduction}

ElectroHydraulic Servo Systems (EHSS) are usually employed for handling heavy loads with fast response [1-2], these systems provide many advantages over electric motors such as high stiffness, high force and torque, self-lubricating properties and low cost. They are usually used in robotic manipulators, aircraft flight-control actuators, hydraulic elevators, active suspension systems and automated manufacturing systems. Its essential components are a pump, a relief valve, a servovalve and a hydraulic actuator. The pump provides a flow of fluid in the system. The relief valve transmits an amount of flow in the pressure line to bound the supply pressure of the system; the servo-valve commands the motion and the pressure of the hydraulic actuator. The hydraulic actuator pilots the load, transmitting the desired displacement, velocity and pressure to the load. The dynamic behavior of hydraulic systems is highly nonlinear which, in turn, cause complexities in the control of such 
systems and make control design for high performance very challenging. Also, the hydraulic parameters may change due to the variation of temperature and the entrapped air in the fluid. Finally, leakages, external load, friction and noise influences result in defies to ensure precise control.

Various control methods have been established to progress the tracking performance of the angular displacement and mitigate the effects of uncertainties in EHSS. In some studies, Feedback controllers using approximate linearization of the nonlinear dynamics were investigated, Standard linear control theory is applied, their performance is only guaranteed in the environs of the operating point. The sliding modes control is applied, it is robust to modeling error with certain conditions, in terms of the uncertain parameters affecting the system, and these methods produce the problem of chattering, due to the switching input. Adaptive feedback linearizing controllers are used to control the velocity and the force of an EHSS system. However, the controller only applies to velocity control in a unidirectional sense.

To resolve the declared problems, a level nonlinear robust controller is created by combining the advantages of Coefficient Diagram Method (CDM) [3] and backstepping method [4-12], CDM-backstepping with nonlinear observer is established to control the EHSS in company of uncertainties where exploiting the robustness of CDM and asymptotic convergence of backstepping. The concept of backstepping is easy. At every step of backstepping, another control Lyapunov function is developed by extension of the control Lyapunov function from the last step by a term [4]. The approach also permits the insertion of other nonlinearities into the control laws for the elimination of undesirable uncertainty. Under the assumption that the states of our system are not accessible, an observer is created to estimate unmeasurable states. Based on the Lyapunov stability analysis, the precision of position control and the convergence of estimating errors can be ensured. From the numerical results, it can be seen that the controlled system has well performance. All system states are bounded and the angular displacement errors of the EHSS asymptotically converge to zero.

CDM-backstepping offers a good alternative to conventional control systems. However, its parameters must be adjusted by trial and error which takes a lot of time for a designer, Ant Colony Optimization (ACO) [15-19] is proposed for this purpose to ensure height performance with optimized parameters control [20-24], it has been successfully applied to various problems [25-28]. In this paper we describe the proposed methodology to design a CDM-backstepping control using the ACO metaheuristic. The controller parameters are designated based on evaluation of objective function ITAE through simulations and the proposed algorithm is viewed as a series of steps, allowing faster optimization and better results.

The paper is organized as follows. In Section 2, the EHSS state space model is presented. In Section 3, the CDM controller is designed for linear system. In Section 4 CDM-backstepping with observer is developed. Then, stability is demonstrated using Lyapunov stability analysis. Section 5 clarifies the concepts of ACO approach. In Section 6, the numerical results are examined to appear the effectiveness of the proposed control scheme; finally conclusions are presented in Section 7. In Appendix are given actual parameters of the EHSS. 


\section{EHSS state space model}

The EHSS model is designated by the next fourth order nonlinear state-space model:

$$
\left\{\begin{array}{l}
\dot{x}=A x+g(x)+B_{\mathrm{c}} u, \\
y=C x,
\end{array}\right.
$$

with

$$
x=\left[\begin{array}{l}
x_{1} \\
x_{2} \\
x_{3} \\
x_{4}
\end{array}\right], A=\left[\begin{array}{cccc}
0 & 1 & 0 & 0 \\
0 & a_{1} & a_{2} & 0 \\
0 & a_{3} & a_{4} & 0 \\
0 & 0 & 0 & a_{5}
\end{array}\right], B_{\mathrm{c}}=\left[\begin{array}{c}
0 \\
0 \\
0 \\
a_{6}
\end{array}\right], C^{\mathrm{T}}=\left[\begin{array}{l}
1 \\
0 \\
0 \\
0
\end{array}\right], g(x)=\left[\begin{array}{c}
0 \\
b_{1}\left(x_{2}\right) \\
b_{2}\left(x_{3}, x_{4}\right) x_{4} \\
0
\end{array}\right],
$$

where $x_{1}(t)$ is the angular displacement; $x_{2}(t)$ is the angular velocity; $x_{3}(t)$ is the motor pressure difference due to the load; $x_{4}(t)$ is the servovalve opening area; $u(t)$ is the control input; $y(t)$ is the system output. The parameters $a_{i}$ and $b_{i}$ are set as

$$
\begin{gathered}
a_{1}=-B / J, a_{2}=D_{\mathrm{m}} / J, a_{3}=-4 \sigma D_{\mathrm{m}} / V_{\mathrm{m}}, a_{4}=-4 \sigma C_{\mathrm{sm}} / V_{\mathrm{m}}, a_{5}=-1 / v, a_{6}=K / v, \\
b_{1}\left(x_{2}\right)=\left(-T_{\mathrm{f}} \operatorname{sgn}\left(x_{2}(t)\right)+T_{I}\right) / J, \\
b_{2}\left(x_{3}, x_{4}\right)=\left(-4 \sigma C_{d} /\left(V_{\mathrm{m}} \sqrt{\rho}\right)\right) \sqrt{P_{s}-\operatorname{sgn}\left(x_{4}(t)\right) x_{3}(t)},
\end{gathered}
$$

where $I$ is the total inertia of the motor; $D_{\mathrm{m}}$ is the total oil volume in the two chambers of the actuator; $B$ is the flow discharge coefficient; $T_{\mathrm{f}}$ is the Coulomb friction coefficient; $T_{1}$ is the load torque; $\sigma$ is the fluid bulk-modulus; $V_{\mathrm{m}}$ is the total oil volume in the two chambers of the actuator; $C_{\mathrm{d}}$ is the flow discharge coefficient, $\rho$ is the fluid mass density; $C_{\mathrm{sm}}$ is the leakage coefficient; $P_{\mathrm{s}}$ is the supply pressure; $K$ is the servovalve amplifier gain and $v$ is the servovalve time constant.

\section{CDM control design}

CDM control is an algebraic method with polynomial form, it permit designing the controller under the requirements of stability, time domain performance and robustness. The performance specification, equivalent time constant and stability index are definite in the closed loop transfer function and form a relationship to the controller parameters algebraically [3]. The output of the controlled is expressed as

$$
y=\frac{N(s) F(s)}{P(s)} r+\frac{A(s) N(s)}{P(s)} d,
$$

where $y$ is the output; $r$ is the reference input and $d$ is the external disturbance signal; $N(s)$ and $D(s)$ are the numerator and the denominator of the transfer function of the system, respectively; $A(s)$ is the denominator polynomial of the controller transfer function, while $F(s)$ and $B(s)$ are named the reference numerator (pre-filter) and the feedback numerator polynomials of the controller transfer function.

As well, $P(s)$ is the characteristic polynomial [3] and is specified as 
(3) $P(s)=D(s) A(s)+N(s) B(s)=\sum_{i=0}^{n} \mu_{i} s^{i}=\mu_{0}\left[\left\{\sum_{i=2}^{n}\left(\prod_{j=1}^{i-1} \frac{1}{\gamma_{i-j}^{j}}\left(T_{0} s\right)^{i}\right)\right\}+T_{0} s+1\right]$,

where $A(s)=\sum_{i=0}^{n} l_{i} s^{i}$ and $B(s)=\sum_{i=0}^{n} r_{i} s^{i}$, the equivalent time constant $T_{0}=\mu_{1} / \mu_{0}$ indicate the time response speed, also the stability indices $\gamma_{i}=\mu_{i}^{2} / \mu_{i-1} \mu_{i+1}$ for $i=1$ to $n-1$, give the stability and the waveform of the time response [3]. The settling time $t_{\mathrm{s}}$ and the equivalent time constant $T_{0}$ are related as $T_{0}=t_{\mathrm{s}} /(2.5 \sim 3)$, with $\gamma_{1}=2.5, \gamma_{i}=2, i=2 \sim(n-1), \gamma_{0}=\gamma_{n}=\infty$. The settling time and the time constant can be modified to provide the needed performance, therefore $\gamma_{i}>1.5$ for all $i=1 \sim(n-1)$. Finally the pre-filter $F(s)=\left.P(s)\right|_{s=0} / N(s)$ is used to decrease the steady state error to zero.

\section{Nonlinear observer based on CDM-backstepping}

In this section, we present the main idea to design a nonlinear observer for the considered system characterized by the local Lipschitz condition, an observer-based CDM-backstepping control method is studied to deal with the angular displacement control problematic, while the angular displacement is the only measured signal.

To offer the nonlinear observer, the Lipschitz condition on the term $g(x)$ given in (1) is in a closed-bounded region $\Omega$, with $\left\|g\left(q_{1}\right)-g\left(q_{2}\right)\right\| \leq \kappa\left\|q_{1}-q_{2}\right\|, q_{1}, q_{2} \in \Omega$ and $\kappa$ is the constant Lipschitz.

The nonlinear observer of the system given by (1) has the following form [13]

$$
\left\{\begin{array}{l}
\hat{\dot{x}}=A \hat{x}+g(\hat{x})+B_{\mathrm{c}} u+H(y-\hat{y}), \\
\hat{y}=C \hat{x}
\end{array}\right.
$$

where $H=\left(\begin{array}{llll}h_{1} & h_{2} & h_{3} & h_{4}\end{array}\right)$ is the observer gain. Describe the estimation error by $e_{\mathrm{o}}=x-\hat{x}$. Consequently, the dynamics of error is specified as

$$
\dot{e}_{\mathrm{o}}=(A-H C) e_{\mathrm{o}}+g(x)-g(\hat{x})=A_{\mathrm{o}} e_{\mathrm{o}}+g(x)-g(\hat{x}) .
$$

Since the pair $(A, C)$ is detectable [13], a stabilization observer gain $H$ is acquired such that the closed-loop system matrix $A_{0}=(A-H C)$ is Hurwitz. There are two symmetric positive definite matrices $P$ and $Q$ with $A_{0}{ }^{\mathrm{T}} P+P A_{0}=-Q$.

Consider the Lyapunov function candidate $V_{\mathrm{o}}=e_{\mathrm{o}}^{\mathrm{T}} P e_{\mathrm{o}}$ [14], its derivative is

$$
\dot{V}_{\mathrm{o}}=e_{\mathrm{o}}^{\mathrm{T}}\left(A_{\mathrm{o}}^{\mathrm{T}} P+P A_{\mathrm{o}}\right) e_{\mathrm{o}}+2 e_{\mathrm{o}}^{\mathrm{T}} P(g(x)-g(\hat{x}))=
$$

$$
=-e_{\mathrm{o}}{ }^{\mathrm{T}} Q e_{\mathrm{o}}+2 e_{\mathrm{o}}{ }^{\mathrm{T}} P(g(x)-g(\hat{x})) \leq-\left(\varsigma_{\min }(Q)-2 \chi\|P\|\right)\left\|e_{\mathrm{o}}\right\|^{2}=-\omega\left\|e_{\mathrm{o}}\right\|^{2} .
$$

Provided the condition $\omega=\left(\varsigma_{\min }(Q)-2 \chi\|P\|\right) \succ 0$ which is fulfilled by suitable design, the asymptotic convergence of estimation error $e_{o}$ can be guaranteed.

Consider the dynamics of EHSS with the angular displacement 


$$
\left\{\begin{array}{l}
\dot{x}_{1}=x_{2}, \\
\hat{\dot{x}}_{2}=a_{1} \hat{x}_{2}+a_{2} \hat{x}_{3}+b_{1}\left(\hat{x}_{2}\right)+h_{2} e_{\mathrm{o} 1}, \\
\hat{\dot{x}}_{3}=a_{3} \hat{x}_{2}+a_{4} \hat{x}_{3}+b_{2}\left(\hat{x}_{3}, \hat{x}_{4}\right) \hat{x}_{4}+h_{3} e_{\mathrm{o} 1}, \\
\hat{\dot{x}}_{4}=a_{5} \hat{x}_{4}+a_{6} u+h_{4} e_{\mathrm{o} 1} .
\end{array}\right.
$$

Describe the tracking error $z_{1}=x_{1}-x_{d}$ with $x_{d}$ is the desired displacement, then $\dot{z}_{1}=\dot{x}_{1}-\dot{x}_{\mathrm{d}}=\hat{x}_{2}+e_{\mathrm{o} 2}-\dot{x}_{\mathrm{d}}$; The first lyapunov function is $V_{1}=0.5 z_{1}^{2}+V_{\mathrm{o}}$, its derivative is $\dot{V}_{1}=z_{1} \dot{z}_{1}+\dot{V}_{\mathrm{o}}, \dot{V}_{1} \leq z_{1} \dot{z}_{1}-\omega\left\|e_{\mathrm{o}}\right\|^{2}=z_{1}\left(\hat{x}_{2}+e_{\mathrm{o} 2}-\dot{x}_{d}\right)-\omega\left\|e_{\mathrm{o}}\right\|^{2}$.

Tacking $\varphi_{1}=-c_{1} z_{1}+\dot{x}_{\mathrm{d}} \quad$ and $\quad z_{2}=\hat{x}_{2}-\varphi_{1}, \quad$ gives $\dot{V}_{1} \leq z_{1}\left(z_{2}+e_{\mathrm{o} 2}-\dot{x}_{\mathrm{d}}+\varphi_{1}\right)-\omega\left\|e_{\mathrm{o}}\right\|^{2}$, where $e_{\mathrm{o} 2}=x_{2}-\hat{x}_{2}$ and $\hat{x}_{2}$ is considered as the control input, after that describing the stabilizing control law $\varphi_{1}=-c_{1} z_{1}+\dot{x}_{\mathrm{d}}$, where $c_{1}>0$, this guide to the tracking error $z_{2}=\hat{x}_{2}-\varphi_{1}$. Then,

$$
\dot{V}_{1} \leq z_{1} z_{2}-c_{1} z_{1}^{2}+z_{1} e_{\mathrm{o} 2}-\omega\left\|e_{\mathrm{o}}\right\|^{2} \text {. }
$$

By using the generic inequality $z_{1} e_{02} \leq \kappa_{1} z_{1}^{2}+\left(1 / 4 \kappa_{1}\right) e_{02}{ }^{2}$, with $\kappa_{1}>0$, one yields

$$
\begin{aligned}
\dot{V}_{1} & \leq z_{1} z_{2}-\left(c_{1}-\kappa_{1}\right) z_{1}^{2}+\left(1 / 4 \kappa_{1}\right) e_{\mathrm{o} 2}{ }^{2}-\omega\left\|e_{\mathrm{o}}\right\|^{2} \leq \\
& \leq z_{1} z_{2}-\left(c_{1}-\kappa_{1}\right) z_{1}^{2}-\left(\omega-\left(1 / 4 \kappa_{1}\right)\right)\left\|e_{\mathrm{o}}\right\|^{2} .
\end{aligned}
$$

If $c_{1}$ and $\kappa_{1}$ are such that $\omega>1 / 4 \kappa_{1}$ and $c_{1}>\kappa_{1}$, then $\dot{V}_{1} \leq z_{1} z_{2}-\bar{c}_{1} z_{1}^{2}, \bar{c}_{1}>0$.

To deal with the control of the subsystem (7), the state $\hat{x}_{3}$ is manipulated as an independent input. The time derivative of $z_{2}$ is given by

$$
\dot{z}_{2}=\dot{\hat{x}}_{2}-\dot{\varphi}_{1}=a_{1} \hat{x}_{2}+a_{2} \hat{x}_{3}+b_{1}\left(\hat{x}_{2}\right)+h_{2} e_{\mathrm{o} 1}+c_{1}\left(\hat{x}_{2}-\dot{x}_{\mathrm{d}}\right)+c_{1} e_{\mathrm{o} 2}-\ddot{x}_{\mathrm{d}} \text {. }
$$

Select the second Lyapunov function as $V_{2}=V_{1}+0.5 z_{2}^{2}+V_{0}$, its derivative is

$$
\dot{V}_{2}=\dot{V}_{1}+z_{2} \dot{z}_{2}+\dot{V}_{\mathrm{o}} \leq z_{1} z_{2}-\bar{c}_{1} z_{1}^{2}+z_{2} \dot{z}_{2}-\omega\left\|e_{\mathrm{o}}\right\|^{2} .
$$

Replacing (9) into (10) results in

$$
\begin{gathered}
\dot{V}_{2} \leq z_{1} z_{2}-\bar{c}_{1} z_{1}^{2}+z_{2}\left(a_{1} \hat{x}_{2}+a_{2} \hat{x}_{3}+\right. \\
\left.+b_{1}\left(\hat{x}_{2}\right)+h_{2} e_{\mathrm{o} 1}+c_{1}\left(\hat{x}_{2}-\dot{x}_{\mathrm{d}}\right)+c_{2} e_{\mathrm{o} 2}-\ddot{x}_{\mathrm{d}}\right)-\omega\left\|e_{\mathrm{o}}\right\|^{2} .
\end{gathered}
$$

Next, the desired command input $\varphi_{2}$ of $x_{3}$ is selected as

$$
\varphi_{2}=\left(1 / a_{2}\right)\left(-a_{1} \hat{x}_{2}-b_{1}\left(\hat{x}_{2}\right)-h_{2} e_{\mathrm{o} 1}-c_{1}\left(\hat{x}_{2}-\dot{x}_{\mathrm{d}}\right)+\ddot{x}_{\mathrm{d}}-z_{1}-c_{2} z_{2}\right) .
$$

Describe the tracking error $z_{3}=\hat{x}_{3}-\varphi_{2}$, with easy manipulation, one yields $\dot{V}_{2} \leq a_{2} z_{2} z_{3}-\bar{c}_{1} z_{1}^{2}-c_{2} z_{2}^{2}+c_{1} e_{\mathrm{o} 2} z_{2}-\omega\left\|e_{\mathrm{o}}\right\|^{2}$. Using inequality $e_{\mathrm{o} 2} z_{2} \leq \kappa_{2} z_{2}{ }^{2}+\left(e_{\mathrm{o} 2}{ }^{2} / 4 \kappa_{2}\right)$, $\kappa_{2}>0$, the last equation can be reorganized as

$$
\begin{aligned}
\dot{V}_{2} & \leq a_{2} z_{2} z_{3}-\bar{c}_{1} z_{1}^{2}-c_{2} z_{2}^{2}+c_{1} \kappa_{2} z_{2}^{2}+\left(c_{1} / 4 \kappa_{2}\right) e_{\mathrm{o} 2}^{2}-\omega\left\|e_{\mathrm{o}}\right\|^{2} \leq \\
& \leq a_{2} z_{2} z_{3}-\bar{c}_{1} z_{1}^{2}-\left(c_{2}-c_{1} \kappa_{2}\right) z_{2}^{2}-\left(\omega-\left(c_{1} / 4 \kappa_{2}\right)\right)\left\|e_{\mathrm{o}}\right\|^{2} .
\end{aligned}
$$

Tacking $\omega>c_{1} / 4 \kappa_{2}, c_{2}>c_{1} \kappa_{2}$ and $\bar{c}_{2}=c_{2}-c_{1} \kappa_{2}$, It follows $\dot{V}_{2} \leq a_{2} z_{2} z_{3}-\bar{c}_{1} z_{1}^{2}-\bar{c}_{2} z_{2}^{2}$. The time derivative of $\varphi_{2}$ is given as 


$$
\begin{aligned}
\dot{\varphi}_{2}= & \left(1 / a_{2}\right)\left(-a_{1} \dot{\hat{x}}_{2}-\dot{b}_{1}\left(\hat{x}_{2}\right)-h_{2} \dot{\mathrm{e}}_{\mathrm{o} 1}-c_{1}\left(\dot{\hat{x}}_{2}-\ddot{x}_{\mathrm{d}}\right)+\dddot{x}_{\mathrm{d}}-\dot{z}_{1}-c_{2} \dot{z}_{2}\right)= \\
= & \left(1 / a_{2}\right)\left(-a_{1}\left(\hat{x}_{2}+e_{\mathrm{o} 2}-\dot{x}_{\mathrm{d}}\right)-h_{2} \dot{e}_{\mathrm{o} 1}-\left(a_{2}+1\right) e_{\mathrm{o} 2}+\left(a_{1}+1\right) \dot{x}_{\mathrm{d}}-\right. \\
& \left.-\left(c_{1}+c_{2}\right) a_{2} \hat{x}_{3}-\left(c_{1}+c_{2}\right) b_{1}\left(\hat{x}_{2}\right)-\left(c_{1}+c_{2}\right) e_{\mathrm{o} 1}+c_{1} \ddot{x}_{\mathrm{d}}+\dddot{x}\right) .
\end{aligned}
$$

The derivative of $z_{3}$ is $\dot{z}_{3}=\dot{\hat{x}}_{3}-\dot{\varphi}_{2}=a_{3} \hat{x}_{2}+a_{4} \hat{x}_{3}+b_{2}\left(\hat{x}_{3}, \hat{x}_{4}\right)+h_{3} e_{\mathrm{o} 1}-\dot{\varphi}_{2}$.

Select the third Lyapunov function $V_{3}=V_{2}+0.5 z_{3}^{2}+V_{\mathrm{o}}$. Then, it follows that

$$
\dot{V}_{3} \leq a_{2} z_{2} z_{3}-\bar{c}_{1} z_{1}^{2}-c_{2} z_{2}^{2}+z_{3} \dot{z}_{3}-\omega\left\|e_{\mathrm{o}}\right\|^{2}
$$

then

$$
\begin{aligned}
\dot{V}_{3} \leq & a_{2} z_{2} z_{3}-\bar{c}_{1} z_{1}^{2}-\bar{c}_{2} z_{2}^{2}+z_{3}\left(a_{3} \hat{x}_{2}+a_{4} \hat{x}_{3}+\right. \\
& \left.+b_{2}\left(\hat{x}_{3}, \hat{x}_{4}\right) \hat{x}_{4}+h_{3} e_{\mathrm{o} 1}-\dot{\varphi}_{2}\right)-\omega\left\|e_{\mathrm{o}}\right\|^{2} .
\end{aligned}
$$

Let $\varphi_{3}=\left(1 / b_{2}\left(\hat{x}_{3}, \hat{x}_{4}\right)\right)\left(-a_{3} \hat{x}_{2}-a_{4} \hat{x}_{3}-h_{3} e_{\mathrm{o} 1}-a_{2} z_{2}-c_{3} z_{3}+\dot{\varphi}_{2}\right), b_{2}\left(\hat{x}_{3}, \hat{x}_{4}\right) \neq 0$, then $\dot{V}_{3} \leq-\bar{c}_{1} z_{1}^{2}-\bar{c}_{2} z_{2}^{2}-c_{3} z_{3}^{2}+b_{2}\left(\hat{x}_{3}, \hat{x}_{4}\right) z_{3} z_{4}$. Final Lyapunov function is set as

$$
V_{4}=V_{3}+0.5 z_{4}^{2}+V_{\mathrm{o}} \text {. }
$$

Describe the stabilizing control law $\varphi_{3}$ and the tracking error as

$$
z_{4}=\hat{x}_{4}-\varphi_{3} \text {. }
$$

Its time derivative is given as $\dot{z}_{4}=\dot{\hat{x}}_{4}-\dot{\varphi}_{3}$.

Tacking $\zeta=\hat{x}_{4}$ is the auxiliary variable, the control signal is written as follows

$$
a_{\mathrm{o} 0}(\hat{x}) u+a_{\mathrm{o} 1}(\hat{x}) \frac{d u}{d t}=z_{\mathrm{o}}(t),
$$

where

$$
z_{\mathrm{o}}(t)=c_{\mathrm{o} 0}(\hat{x}) \varphi_{3}-b_{\mathrm{o} 0}(\hat{x}) \zeta-b_{\mathrm{o} 1}(\hat{x}) \dot{\zeta},
$$

$a_{o 0}(x), a_{o 1}(x), c_{o 0}(x), b_{o 0}(x)$ and $b_{o 1}(x)$ are nonlinear gains of nonlinear CDM.

Consider the EHSS dynamic given by (1), in closed-loop with the nonlinear CDM control (18) and (19) and suppose that the gains $\delta$ and $c_{\mathrm{c}}$ are such that

$$
\left|c_{\mathrm{c} 0} \delta \operatorname{sign}\left(z_{s}\right) \int_{0}^{t} z_{4}(\theta) d \theta\right| \geq\left|b_{2}\left(\hat{x}_{3}, \hat{x}_{4}\right) z_{3}\right|+\left|h_{\mathrm{o}}(\hat{x})\right| .
$$

The control signal that obliges $z_{4}(t)$ to converge to zero will be defined, let

$$
\left\{\begin{array}{l}
a_{\mathrm{o} 0}(\hat{x})=-k_{\mathrm{o}}(d g(\hat{x})) / d t=0, \\
a_{\mathrm{o} 1}(\hat{x})=-k_{\mathrm{o}} g(\hat{x}) .
\end{array}\right.
$$

With $k_{\mathrm{o}}>0$, then joining (17) with (19) gives $z_{4}=\left(b_{\mathrm{o} 0}{ }^{-1} c_{\mathrm{c}}-1\right) \varphi_{3}-b_{\mathrm{o} 0}{ }^{-1} z_{\mathrm{o}}$ and tacking $c_{\mathrm{o} 0}(x)=b_{\mathrm{o} 0}(x)=c_{\mathrm{c} 0}$, then $z_{\mathrm{o}}=-c_{\mathrm{o} 0} z_{4}$, its second derivative is given as

Combining (18), (19) and (21) gives

$$
\ddot{z}_{\mathrm{o}}(t)=c_{\mathrm{o} 0} \ddot{\varphi}_{3}(t)-c_{\mathrm{o} 0} \ddot{\zeta} \text {. }
$$

$$
\ddot{\zeta}(t)=a_{5} \dot{\zeta}_{4}(t)+k_{c 1} \zeta z_{\mathrm{o}} .
$$

With $k_{\mathrm{c} 1}=k_{\mathrm{c}}^{-1}$, using (22) and (23), gives $\ddot{z}_{\mathrm{o}}(t)=c_{\mathrm{o} 0} \ddot{\varphi}_{3}(t)-c_{\mathrm{o} 0}\left(a_{5} \dot{\zeta}+k_{\mathrm{c} 1} z_{\mathrm{o}}\right)$, or 
$\dot{z}_{\mathrm{o}}(t)=c_{\mathrm{o} 0} \dot{\varphi}_{3}(t)-c_{\mathrm{o} 0}\left(a_{5} \zeta+k_{c 1} \int_{0}^{t} z_{\mathrm{o}}(\theta) d \theta\right)$ and using (16) gives

$$
\dot{z}_{4}(t)=h_{\mathrm{o}}(\hat{x})-k_{\mathrm{o} 2} \int_{0}^{t} z_{4}(\theta) d \theta .
$$

With $k_{\mathrm{o} 2}=c_{\mathrm{o} 0} k_{\mathrm{o} 1}$ and $h_{\mathrm{o}}(\hat{x})=a_{5} \zeta+h_{4} e_{\mathrm{o} 4}-\dot{\varphi}_{3}(t)$, then taking $k_{\mathrm{o} 2}=\delta \operatorname{sign}\left(z_{s}\right)$ and $z_{s}=z_{4} \int_{0}^{t} z_{4}(\theta) d \theta$ gives

$$
\dot{V}_{4}=\dot{V}_{3}+z_{4} \dot{z}_{4}+\dot{V}_{\mathrm{o}} \leq-\bar{c}_{1} z_{1}^{2}-\bar{c}_{2} z_{2}^{2}-c_{3} z_{3}^{2}+b_{2}\left(\hat{x}_{3}, \hat{x}_{4}\right) z_{3} z_{4}+z_{4} \dot{z}_{4}-\omega\left\|e_{\mathrm{o}}\right\|^{2} \leq
$$

$\leq-\bar{c}_{1} z_{1}^{2}-\bar{c}_{2} z_{2}^{2}-c_{3} z_{3}^{2}+b_{2}\left(\hat{x}_{3}, \hat{x}_{4}\right) z_{3} z_{4}+z_{4}\left(a_{5} \hat{x}_{4}+h_{4} e_{\mathrm{o} 1}-\dot{\varphi}_{3}-K_{\mathrm{o} 2} \int_{0}^{t} z_{4}(\theta) d \theta\right)$.

Using (24) and (18), next one has $\dot{V}_{4} \leq-\bar{c}_{1} z_{1}{ }^{2}-\bar{c}_{2} z_{2}{ }^{2}-c_{3} z_{3}{ }^{2}-c_{4} z_{4}{ }^{2}+v(t)$ where $v(t)=z_{4}\left(b_{2}\left(\hat{x}_{3}, \hat{x}_{4}\right) z_{3}+h(\hat{x})-c_{\mathrm{o} 0} \delta z_{s} \operatorname{sgn}\left(z_{s}\right)\right)$, If $v(t)<0$, then the derivative of the final Lyapunov function is $\dot{V}_{4} \leq-\bar{c}_{1} z_{1}{ }^{2}-\bar{c}_{2} z_{2}{ }^{2}-c_{3} z_{3}{ }^{2}-c_{4} z_{4}{ }^{2}$. As a result $\dot{V}_{4} \leq 0$, this designates that the objective of angular displacement control is finished.

In order to accomplish effective disturbance rejection and robustness to uncertainties, it is crucial to find the optimum values $\delta, c_{\mathrm{c}}$ and the observer gain $H$, which are optimized using a global optimization algorithm as shown in Fig. 1.

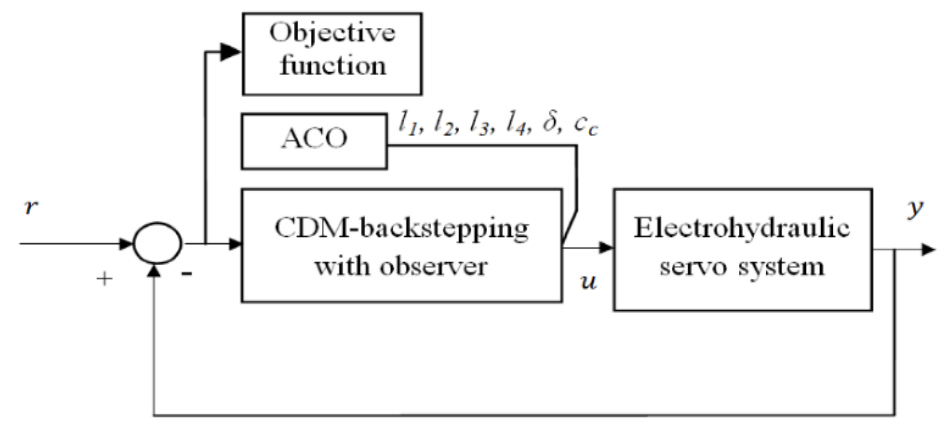

Fig. 1. Block-diagram of CDM-backstepping with observer for EHSS

The performance criteria used to represent the performance of the controller and the observer is the integral of time multiplied by absolute error $J=\int_{0}^{\infty} t|e(t)| d t$, which gives small overshoots, well-damped oscillations and penalizes time while minimizing the error which are extremely suitable for an EHSS problem.

\section{The ACO technique}

ACO is an evolutionary algorithm, proposed by Dorigo and Stützle [15], Dorigo and Blum [16], and Dorigo, Birattari and Stützle [17], for resolving computational problems by finding minimum cost path in a graph from the colony to food as shown in Fig. 2. Really, the ants are directed by pheromone trails and heuristic information to start production of solution in the graph. In artificial ants, 
better paths are found as a result of the global cooperation between ants in the colony [15]. Each ant updates the pheromones deposited to the paths it followed after completing one tour and updates rules; they are driven by a probability rule to choose their tour. When an ant $k$ visited to $i$ nodes and so far constructed the partial solution $S^{P}$, the probability rule for $k$-th at a particular node $i$ to choose the route from node $i$ to node $j$ is specified by

$$
p_{i j}{ }^{k}= \begin{cases}\frac{\left[\tau_{i j}{ }^{\alpha}(n)\right]\left[\eta_{i j}{ }^{\beta}(n)\right]}{\sum_{C_{i i} \in N\left(S^{P}\right)}\left[\tau_{i j}{ }^{\alpha}(n)\right]\left[\eta_{i j}{ }^{\beta}(n)\right]} & \text { if } \quad C_{i j} \in N\left(S^{P}\right), \\ 0 & \text { otherwise, }\end{cases}
$$

where $\tau_{i j}$ and $\eta_{i j}$ are the concentration of pheromone trail associated with the edge and the desirability between the nodes $i$ and $j ; N\left(S^{P}\right)$ and $l$ are set of possible nodes and path that has not been visited by the ant $k$. The positive value $\alpha$ and $\beta$ are the parameters controlling the relative importance of pheromone concentration and desirability respectively for each ant's decision. The ants completes one tour after time and the pheromone concentration change in the trails as $\tau(k+1)_{i j}=(1-\lambda) \tau(k)_{i j}+\Delta \tau_{i j}$. The desirability is selected as $\eta_{i j}=1 / J_{i q}, i=1, \ldots, 6$, and $q=1, \ldots, m$, that is the heuristic information which is inversely proportional to the objective function associated to one node by considering all the rest of parameters values are equals to zeros. Where $0<\lambda<1$ denotes the rate of pheromone evaporation, $\tau(k+1)_{i j}$ and $\tau(k)_{i j}$ illustrates the pheromone concentration of tour at moment $k+1$ and $k$. The increment $\Delta \tau_{i j}$ is the pheromone deposited in the trails with $\Delta \tau(k)_{i j}=\sum_{k=1}^{m} \Delta \tau(k)_{i j}{ }^{k}$. Where, $m$ is the number of ants and $\Delta \tau(k)_{i j}{ }^{k}$ is the additional pheromone laid on the path $(i, j)$ by the ant $k$ at the end of iteration $k$, it is given as

$$
\Delta \tau(k)_{i j}{ }^{k}= \begin{cases}Q / J_{i q}{ }^{k} & \text { if the edge }(i, j) \in \text { globally best tour, } \\ 0 & \text { otherwise, }\end{cases}
$$

where $Q$ is a constant parameter and $J_{i q}{ }^{k}$ is the value of the objective function for the ant $k$.

Fig. 2 provides a graphical representation of the ACO approach of the proposed controller, the controller parameters $l_{1}, l_{2}, l_{3}, l_{4}, \delta$ and $c_{\mathrm{c}}$ are organised in six column lists where each parameter value is designated by $m$ valid digits (nodes), therefore, only one node denotes the optimum solution values of the controller's parameters. Initially, all ants are distributed from random nodes of the construction graph and the pheromone levels $\tau_{i j}$ associated with each arc $(i, j)$ are set to an initial value, in each iteration all ants generate a probability vector based on the pheromone and heuristic values. Founded on this probability vector, at each step ants join one still unvisited nodes to their partial tour. The solution construction finishes once all nodes have been visited. 


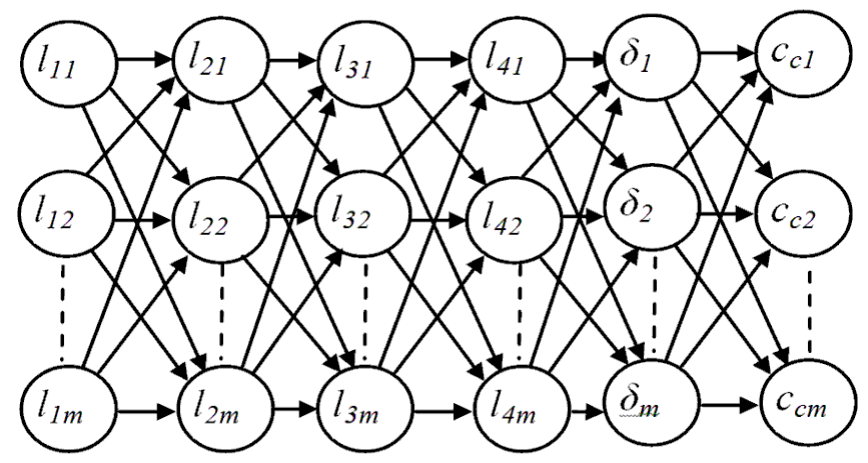

Fig. 2. ACO graph

\section{Simulation results}

The simulation is carried out on EHSS to evaluate the validity of the angular displacement control for a constant and sinusoidal reference. This is illustrated in Fig. 3 to Fig. 10 and shows the performance of the observer as well as the controller in asymptotic convergence of the estimation and tracking errors. The simulation is achieved with the initial state $x(0)=\left[\begin{array}{llll}0 & 0 & 0 & 0\end{array}\right]$ and $z(0)=\left[\begin{array}{llll}0.4 & 0 & 0 & 0\end{array}\right]$, when the parameters of the ACO are: Ant number $=15, Q=120$, Nodes in each vector $=100$, the Maximum tour $=100$, the rate of pheromone evaporation $\lambda=0.6$, relative importance of pheromone concentration and desirability $\alpha=1, \beta=1$, respectively.

\subsection{Test one: Ideal case}

In the first simulations, non-zero initial errors are used, no uncertainties, no external disturbances, and no sensor noises. The EHSS is controlled to track a constant reference about a $1 \mathrm{rad}$ in amplitude with the time span of $2.5 \mathrm{~s}$. The controller performance is presented in Fig. 5, Fig. 6, Fig. 7 and exposes the time angular displacement of tracking and observer errors, its shows that ACO can reduce the settling time and control effort, thereby indicating its effectiveness.

Table 1. The compare of each algorithm performance

\begin{tabular}{|c|c|c|c|c|c|}
\hline Algorithms & $\delta$ & $c_{\mathrm{c}}$ & $H$ & $t_{\mathrm{s}}(s)$ & $J$ \\
\hline Traditional CDM-backstepping & 35 & 0.25 & {$\left[\begin{array}{llll}25 & 5 & 3 & 0.8\end{array}\right]$} & 0.14 & $8.1 \times 10^{-5}$ \\
\hline ACO/CDM-backstepping & 31 & 0.22 & {$\left[\begin{array}{lll}22 & 42 & 0.5\end{array}\right]$} & 0.105 & $1.05 \times 10^{-5}$ \\
\hline
\end{tabular}

\subsection{Test two: Uncertainties, external disturbance and noise}

To verify the robustness of the suggested controller, the hydraulic parameters are assumed to be $10 \%$ of uncertainties in total leakage coefficient and effective oil volume in system, the supply pressure, $P_{\mathrm{s}}$ is reduced by $80 \%$ of its nominal value of 8.73 MPA between $t=0.5 \mathrm{~s}$ and $t=1 \mathrm{~s}$ and at $t=1 \mathrm{~s}, P_{\mathrm{s}}$ varies in a sinusoidal form as shown in Fig. 3. The fluid bulk-modulus, $\sigma$, by $25 \%$ between the moment $t=1.5 \mathrm{~s}$ to $t=2 \mathrm{~s}$ as shown in Fig. 4 and in the presence of a sinusoidal external load disturbance of amplitude 5 N.m, also we introduce $5 \%$ of random noise. The simulation results for a sinusoidal reference in the presence of these uncertainties are presented in 
Fig. 8, Fig. 9, Fig. 10, and show the estimated angular displacements and control inputs. Fig. 8 shows time response of the process output with comparison of proposed and traditional controllers. It can be seen that the proposed controller produces much better performances in terms of robustness of parameters settings. It can exhibit time response without overshoot and reduces the settling time, this evidence of its swiftness, which is a great advantage in control.

Table 1 provides a summary of the performance comparison between $\mathrm{ACO} / \mathrm{CDM}$-backstepping and traditional CDM-backstepping algorithms.

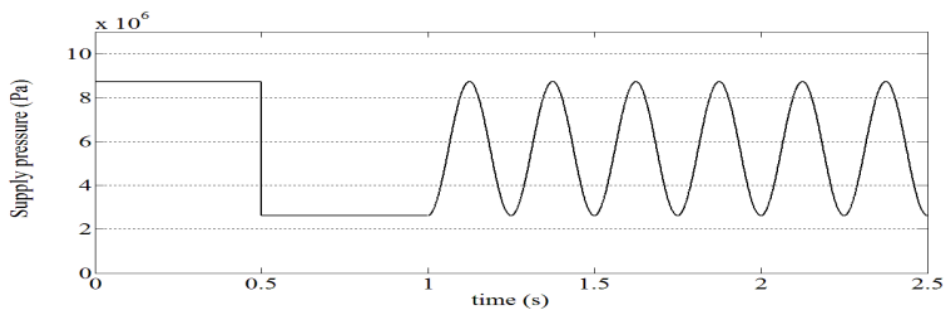

Fig. 3. Simulation of uncertainty in the supply pressure

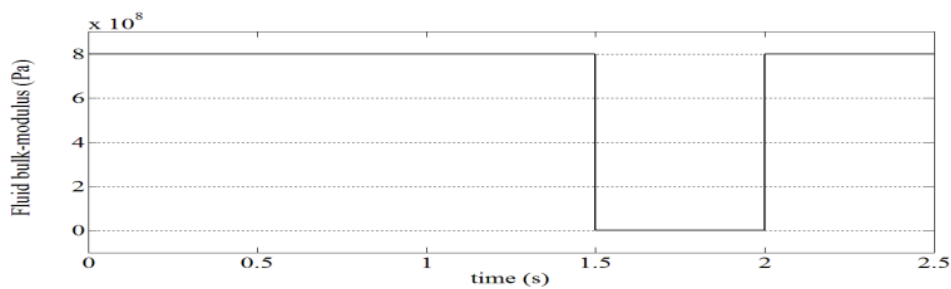

Fig. 4. Simulation of the uncertainty in fluid bulk-modulus

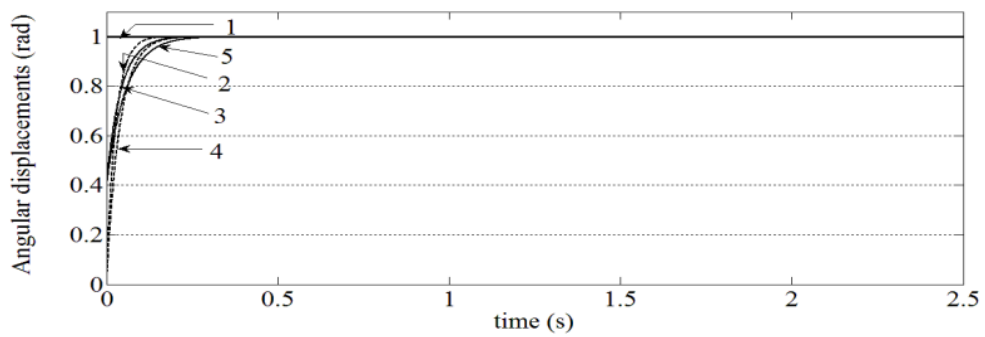

Fig. 5. Test one, Angular displacement: 1) Reference ACO/CDM-backstepping;

2) Actual displacement; 3) Estimated displacement Traditional CDM-backstepping;

4) Actual displacement; 5) Estimated displacement

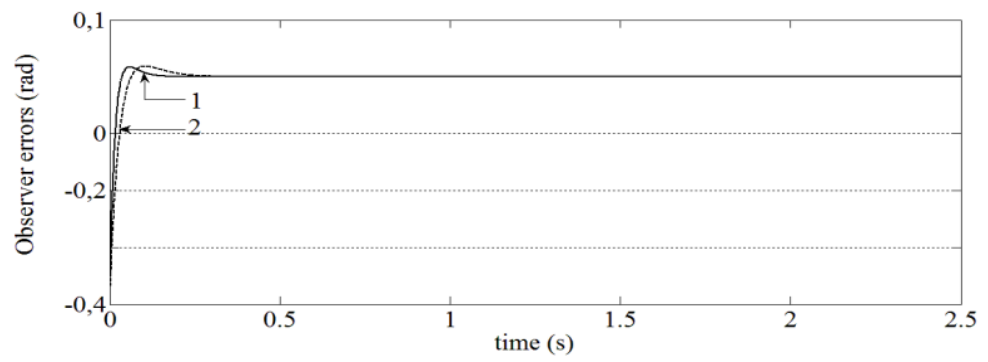

Fig. 6. Test one, Observer error for displacements: 1) ACO/CDM-backstepping;

2) Traditional CDM-backstepping 


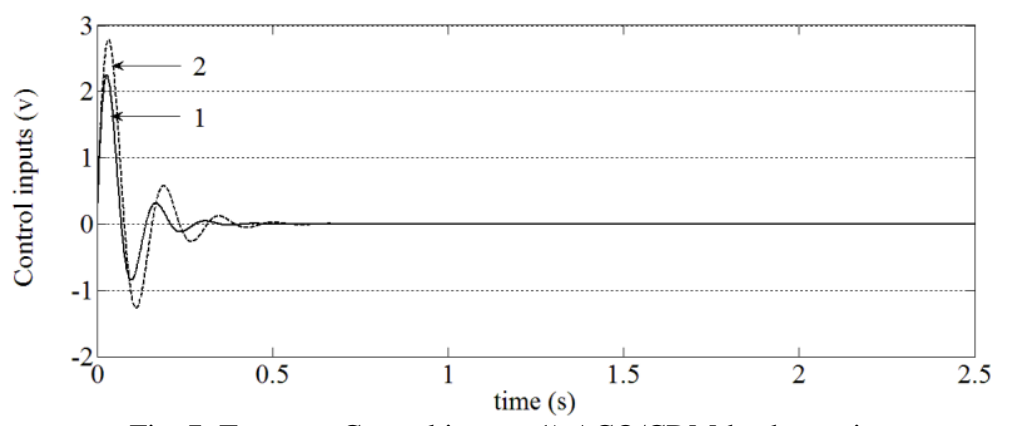

Fig. 7. Test one, Control inputs: 1) ACO/CDM-backstepping;

2) Traditional CDM-backstepping

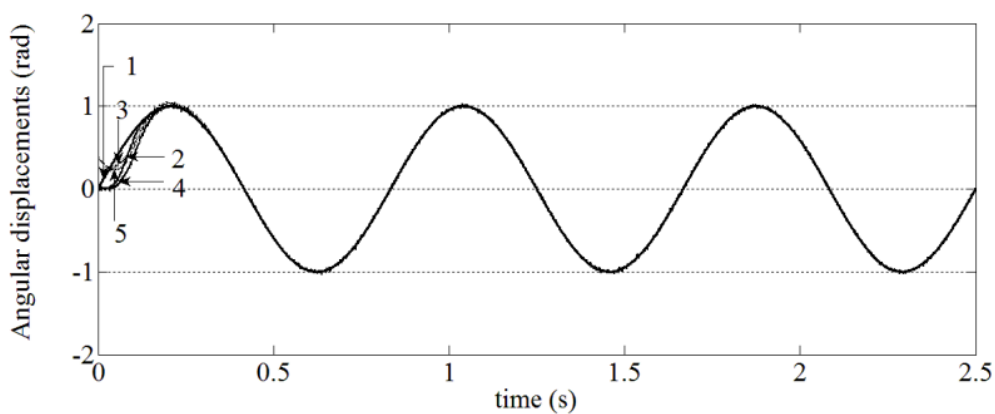

Fig. 8. Test one, Angular displacement: 1) Reference ACO/CDM-backstepping; 2) Actual displacement; 3) Estimated displacement Traditional CDM-backstepping; 4) Actual displacement; 5) Estimated displacement

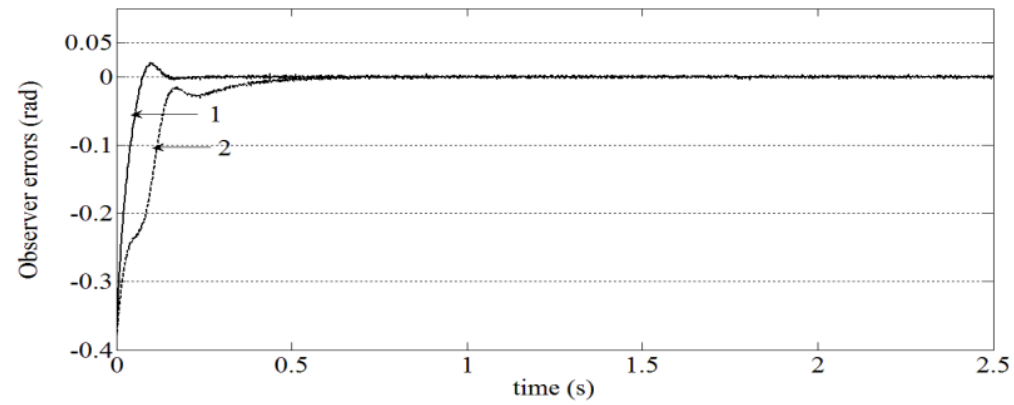

Fig. 9. Test two, Observer error for displacements: 1) ACO/CDM-backstepping; 2) Traditional CDM-backstepping

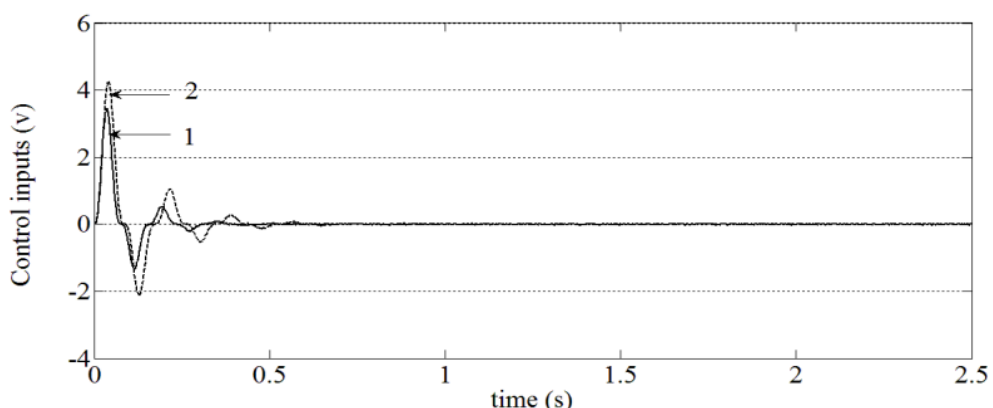

Fig. 10. Test two, Control inputs: 1) ACO/CDM-backstepping;

2) Traditional CDM-backstepping 


\section{Conclusion}

The present work addressed an intelligent motion control strategy that makes possible the introduction of the ACO approach for tuning the parameters of the robust controller CDM-backstepping with observer for position tracking of EHSS, with supply pressure uncertainty. The asymptotic stability of the closed-loop system is demonstrated by utilizing the Lyapunov theorem. The simulation results clearly demonstrate that the proposed strategy is a significant optimization tool for the electrohydraulic servo system, to guarantees stability, ensures tracking with fast response, high precision strong robustness to disturbance and uncertainties, so that it may be useful for the position tracking of high-performance of industrial system.

\section{References}

1. L i, X., X. C h e n, C. Z h o u. Combined Observer-Controller Synthesis for Electro-Hydraulic Servo System with Modeling Uncertainties and Partial State Feedback. - Journal of the Franklin Institute, Vol. 355, 2018, pp. 5893-5911.

2. B a h r a mi, M., M. N a r a g h i, M. Z a r e i n e j a d. Adaptive Super-Twisting Observer for Fault Reconstruction in Electro-Hydraulic Systems. - ISA Transactions, Vol. 76, 2018, pp. 235-245.

3. E r k a n, K., B. C. Y a 1 c 1 n, M. G a r i p. Three-Axis Gap Clearance I-PD Controller Design Based on Coefficient Diagram Method for 4-Pole Hybrid Electromagnet. - Automatika, Vol. 58, 2017, No 2, pp. 147-167.

4. Arsal a n, M., R. Iftikh ar, I. Ah ma d, A. H a s a n, K. S ab a h at, A. J a veri a. MPPT for Photovoltaic System Using Nonlinear Backstepping Controller with Integral Action. - Solar Energy, Vol. 170, 2018, pp. 192-200.

5. A n d r a d e, G. A. D., R. V a z q u e z, D. J. P a g a n o. Backstepping Stabilization of a Linearized ODE-PDE Rijke Tube Model. - Automatica, Vol. 96, 2018, pp. 98-109.

6. L i u, Y., X. L i u, Y. J in g, S. Z h o u. Adaptive Backstepping $\mathrm{H}_{\infty}$ Tracking Control with Prescribed Performance for Internet Congestion. - ISA Transactions, Vol. 72, 2018, pp. 92-99.

7. Witk ow ska, A., R. Śmi e r z chalski. Adaptive Dynamic Control Allocation for Dynamic Positioning of Marine Vessel Based on Backstepping Method and Sequential Quadratic Programming. - Ocean Engineering, Vol. 163, 2018, pp. 570-582.

8. V i j a y, M., D. J e n a. Backstepping Terminal Sliding Mode Control of Robot Manipulator Using Radial Basis Functional Neural Networks. - Computers and Electrical Engineering, Vol. 67, 2018, pp. 690-707.

9. G u o, F., Y. L i u, Y. W u, F. L u o. Observer-Based Backstepping Boundary Control for a Flexible Riser System. - Mechanical Systems and Signal Processing, Vol. 111, 2018, pp. 314-330

10. H u, J., J. H u a n g, Z. G a o, H. G u. Position Tracking Control of a Helicopter in Ground Effect Using Nonlinear Disturbance Observer-Based Incremental Backstepping Approach. Aerospace Science and Technology, Vol. 81, 2018, pp. 167-178.

11. J i, N., J. L i u. Vibration Control for a Flexible Satellite with Input Constraint Based On Nussbaum Function via Backstepping Method. - Aerospace Science and Technology, Vol. 77, 2018, pp. 563-572.

12. He r z i g, N., R. M or e a u, T. R e d a r c e, F. A b r y, X. B r u n. Nonlinear Position and Stiffness Backstepping Controller for a Two Degrees of Freedom Pneumatic Robot. - Control Engineering Practice, Vol. 73, 2018, pp. 26-39.

13. Malikov, A. I. State Observer Synthesis by Measurement Results for Nonlinear Lipschitz Systems with Uncertain Disturbances. - Automation and Remote Control, Vol. 78, 2017, No 5, pp. 782-797.

14. C u i, M., H, Li u., W. Li u. Extended State Observer-Based Adaptive Control for a Class of Nonlinear System with Uncertainties. - Control and Intelligent Systems, Vol. 45, 2017, No 3, pp. 132-141. 
15. D o r i g o, M., T. S t ü t z l e. Ant Colony Optimization. Cambridge, MIT Press, 2004.

16. D o r i g o, M., C. B 1 u m. Ant Colony Optimization Theory: A Survey. - Theoretical Computer Science Vol. 344, 2005, pp. 243-278.

17. Dorigo, M., M. B irattari, T. Stützle. Ant Colony Optimization: Artificial Ants as a Computational Intelligence Technique. - IEEE Computational Intelligence Magazine, Vol. 1, 2006, No 4, pp. 28-39.

18. S o c h a, K., M. D o r i g o. Ant Colony Optimization for Continuous Domains - European Journal of Operational Research. Vol. 185, 2008, No 3, pp. 1155-1173.

19. B i r a t t a r i, M., P. P e 11 e g r i n i., M. D o r i g o. On the Invariance of Ant Colony Optimization. - IEEE Transactions on Evolutionary Computation, Vol. 11, 2007, No 6, pp. 732-742.

20. X i a n g s on g, K., C. X u r u i, G. Ji a n s h e n g. PID Controller Design Based on Radial Basis Function Neural Networks for the Steam Generator Level Control. - Cybernetics and Information Technologies, Vol. 16, 2016, No 5, pp. 15-26.

21. K h e r a b a di, H. A., S. E. M o od, M. M. J a vidi. Mutation: A New Operator in Gravitational Search Algorithm Using Fuzzy Controller - Cybernetics and Information Technologies Vol. 17. 2017, No 1, pp. 72-86.

22. Roeva, O., T. Slavov, S. Fidanova. Population-Based vs. Single Point Search MetaHeuristics for a PID Controller Tuning. - In: Handbook of Research on Novel Soft Computing Intelligent Algorithms: Theory and Practical Applications. P. Vasant, Ed. Vol. 1 and 2. IGI Global, 2014. Web 8 May 2013, pp. 200-233. DOI:10.4018/978-1-4666-4450-2, ISBN13: 9781466644502, ISBN10: 1466644508, EISBN13: 9781466644519.

23. Roeva, O., T. Slavov. PID Controller Tuning Based on Metaheuristic Algorithms for Bioprocess Control - Biotechnology \& Biotechnological Equipment, Vol. 26, 2014, No 5, pp. 3267-3277.

24. Roeva, O., T. Slavov. A New Hybrid GA-FA Tuning of PID Controller for Glucose Concentration Control - Recent Advances in Computational Optimization, Vol. 470, 2013. pp. $155-168$.

25. Li, J., Z. Zhongqiang, W. Y anwei, W. Xia ojing, H. Guihua, L. Shiming, D. F at ag. Research on Electro-hydraulic Force Servo System and its Control Strategy Considering Transmission Clearance and Friction. - Acta Technica, Vol. 61, 2017, No 4, pp. 207-218.

26. K u m a r, P. M., U. D. G a ndh i, G. M a n o gar a n, R. S und ar a se k a r, N. C h i l a m k u rti, R. V a r a th a r aj a n. Ant Colony Optimization Algorithm with Internet of Vehicles for Intelligent Traffic Control System. - Computer Networks, Vol. 144, 2018, pp. 154-162.

27. M o k h t a r i, Y., D R e k i o u a. High Performance of Maximum Power Point Tracking Using Ant Colony Algorithm in Wind Turbine. - Renewable Energy, Vol. 126, 2018, pp. 1055-1063.

28. M o h a m m e d, A. Modern Optimization Techniques for PID Parameters of Electrohydraulic Servo Control System. - International Journal on Recent and Innovation Trends in Computing and Communication, Vol. 5, 2017, No 3, pp. 71-79.

\section{Appendix}

\section{Actual parameters of the EHSS}

$I=1.3$ N.m.s s $^{2} D_{\mathrm{m}}=2.59 \times 10^{-6} \mathrm{~m} / \mathrm{rad}, B=10.36$ N.m.s, $V_{\mathrm{m}}=2.4 \times 10^{-4} \mathrm{~m}^{3}$, $C_{\mathrm{d}}=0.61, T_{\mathrm{f}}=322.5$ N.m, $T_{1}=0$ N.m, $4 \sigma / V_{\mathrm{m}}=1.89 \times 10^{13} \mathrm{~Pa} / \mathrm{m}^{3}, \rho=874 \mathrm{~kg} / \mathrm{m}^{3}$, $v=0.0106 \mathrm{~s}, K=1.54 \times 10^{-6} \mathrm{~m}^{2} / \mathrm{mA}, C_{\mathrm{sm}}=6.34 \times 10^{-14} \mathrm{~m}^{5} /(\mathrm{N} . \mathrm{s}), P_{\mathrm{s}}=8.73 \times 10^{6} \mathrm{~Pa}$.

Received: 11.08.2018; Second Version: 10.11.2018; Third Version: 24.11.2018; Accepted: 20.12.2018 